\title{
Subcarrier Clustering for MISO-OFDM Channels with Quantized Beamforming
}

\author{
Kritsada Mamat and Wiroonsak Santipach \\ Department of Electrical Engineering \\ Faculty of Engineering, Kasetsart University \\ Bangkok, Thailand 10900 \\ Email: $\{\mathrm{g} 5317500192$,wiroonsak.s\}@ku.ac.th
}

\begin{abstract}
We consider a transmit beamforming for an OFDM channel with multiple transmit antennas and single receive antenna. With channel information, a receiver selects and quantizes transmit beamforming vector for each subcarrier. The quantized beamformers are then relayed to the transmitter via a ratelimited feedback channel. We propose to reduce the required number of feedback bits by applying a common transmit beamformer for a cluster of adjacent subcarriers. The sum capacity over all subcarriers depends on a cluster size, the number of feedback bits, and the number of channel taps. Approximation of the optimal cluster size that maximizes the sum rates is derived and is shown to predict simulation results very well. Numerical results show that operating with the optimal cluster size can achieve significant performance gain.
\end{abstract}

\section{INTRODUCTION}

Orthogonal frequency-division multiple access (OFDM) delivers a high data rate for today's ever increasing demand and eliminates inter-symbol interference (ISI). Equipping transmitter and receiver with multiple antennas has been shown to increase the capacity [1] and link reliability. Thus, many current and future wireless standards are based on multiple-antenna OFDM [2]. In this work, we consider an OFDM channel with multiple transmit antennas and single receive antenna. For a multiple-input single-output (MISO) channel, beamforming is a simple and effective method to increase an achievable rate [3]. For beamforming, a transmit waveform is transmitted in a direction of the strongest channel mode by adjusting transmit antenna coefficients. However, this technique requires channel information at the transmitter.

Obtaining channel information at a receiver is achieved by sending pilot signal. The transmitter, on the other hand, cannot estimate the channel by itself, especially in frequency-division duplex (FDD) and has to rely on the receiver for channel information via a feedback channel. With channel information, the receiver determines the optimal transmit beamformer and sends it back to the transmitter. Since feedback channel is normally rated limited, the transmit beamforming vector need to be quantized. Many quantization schemes and codebooks have been proposed and analyzed [4]-[7, see references therein].

This work was supported by the 2010 Telecommunications Research and Industrial Development Institute (TRIDI) scholarship and Kasetsart University Research and Development Institute (KURDI) under the FY2012 Kasetsart University research grant.
The corresponding system performance was shown to depend on codebook design and available feedback bits.

Majority of the previous work on quantized beamforming focus on a narrowband system. Here we consider a wideband channel, which OFDM transforms into several parallel subchannels or subcarriers. If the transmit beamforming vector, which consists of transmit antenna coefficients, for each subcarrier is quantized separately, the total number of feedback bits required can be prohibitively high. References [8]-[10] have proposed methods to reduce the number of feedback bits in OFDM system with multiple antennas. All proposed to use the same or similar beamforming vectors for a group or cluster of adjacent subcarriers since subcarriers are highly correlated in a frequency-selective channel. However, none has analyzed the optimal cluster size and the associated performance. Clustering of subcarriers in OFDM was also applied to select modulation schemes [11].

In this work, we quantize the transmit beamforming vector of each subcarrier with a random vector quantization (RVQ) codebook. RVQ uses a simple codebook, which contains independent isotropically distributed vectors and was shown to perform close to the optimum codebook [6], [12]. To reduce amount of feedback, we propose to group nearby subcarriers into clusters and use the same quantized beamforming vector for all subcarriers in the same cluster. The common beamforming vector for a cluster is chosen to be the quantized beamformer of the subcarrier in the middle or next to the middle in the cluster. We determine the optimal cluster size that maximizes the sum capacity. This has not been investigated by others and is a very valuable and useful parameter. We show that the optimal cluster size is a function of the number of channel taps and available feedback bits and that operating with the optimal cluster size gives a significant performance gain

\section{Channel Model}

We consider a point-to-point discrete-time multiple-input single-output (MISO)-OFDM channel with $N$ subcarriers. A transmitter is equipped with $M$ antennas while a receiver is equipped with single antenna. We assume that transmit antennas are placed sufficiently far apart that they are independent. For each transmit-receive antenna pair, a transmitted signal propagates through a frequency-selective Rayleigh 
fading channel with order $L$. We denote a channel impulse response of the $m$ th transmit-receive antenna pair by an $L \times 1$ vector $\boldsymbol{h}^{m}=\left[\begin{array}{llll}h_{0}^{m} & h_{1}^{m} & \cdots & h_{L-1}^{m}\end{array}\right]^{T}$. Each channel tap $h_{l}^{m}$ is an independent complex Gaussian random variable with zero mean and variance $\sigma_{m, l}^{2}$. Assuming a uniform power delay profile for all antenna pairs, $\sigma_{m, l}^{2}=\frac{1}{L}$ for $\forall l$ and $\forall m$. Thus,

$$
\sum_{l=0}^{L-1} \sigma_{m, l}^{2}=1
$$

A frequency response at the $n$th subcarrier is given by a discrete Fourier transform of the $L$-tap channel as follows

$$
H_{n}^{m}=\boldsymbol{h}_{m}^{T} \boldsymbol{D}_{n}=\sum_{l=0}^{L-1} h_{l}^{m} \mathrm{e}^{\frac{-j 2 \pi l n}{N}}
$$

where $\boldsymbol{D}_{n}=\left[\begin{array}{lllll}1 & \mathrm{e}^{\frac{-j 2 \pi n}{N}} & \mathrm{e}^{\frac{-j 2 \pi 2 n}{N}} & \cdots & \mathrm{e}^{\frac{-j 2 \pi(L-1) n}{N}}\end{array}\right]^{T}$.

Assuming a transmit beamforming or a rank-one precoding, the received signal on the $n$th subcarrier is given by

$$
r_{n}=\boldsymbol{H}_{n}^{\dagger} \boldsymbol{v}_{n} x_{n}+z_{n}
$$

where the $M \times 1$ channel vector for the $n$th subcarrier $\boldsymbol{H}_{n}=\left[\begin{array}{llll}H_{n}^{1} & H_{n}^{2} & \cdots & H_{n}^{M}\end{array}\right]^{T}, \boldsymbol{v}_{n}$ is an $M \times 1$ unit-norm beamforming vector, $x_{n}$ is a transmitted symbol with zero mean and unit variance, and $z_{n}$ is an additive white Gaussian noise with zero mean and variance $\sigma_{z}^{2}$.

Thus, the associated sum capacity over all subcarriers is given by

$$
C=\sum_{n=0}^{N-1} E_{\boldsymbol{H}_{n}}\left[\log \left(1+\rho \boldsymbol{v}_{n}^{\dagger} \boldsymbol{H}_{n} \boldsymbol{H}_{n}^{\dagger} \boldsymbol{v}_{n}\right)\right]
$$

where the expectation is over distribution of $\boldsymbol{H}_{n}$. We assume uniform power allocation for all subcarriers and hence, the background signal-to-noise ratio (SNR) for each subcarrier $\rho=1 / \sigma_{z}^{2}$. From (4), we note that the sum capacity is a function of a set of beamforming vectors $\left\{\boldsymbol{v}_{0}, \boldsymbol{v}_{1}, \ldots, \boldsymbol{v}_{N-1}\right\}$.

The receiver with perfect channel information can optimize the sum capacity over transmit beamforming vectors. For the $n$th subcarrier, the optimal $\boldsymbol{v}_{n}$ that maximizes the rate is the normalized channel vector $\boldsymbol{H}_{n} /\left\|\boldsymbol{H}_{n}\right\|$. With unlimited feedback, the receiver can relay the optimal beamforming vector to the transmitter without quantization. Practically, the feedback channel between the receiver and the transmitter is rate-limited. Thus, quantization of the optimized beamforming vector is required. Here we apply a random vector quantization (RVQ) codebook whose entry is a independent isotropically distributed vector to quantize a transmit beamforming vector. RVQ is simple, but was shown to perform close to the optimum codebook [6], [12], [13].

With available $B_{n}$ feedback bits and the RVQ codebook denoted by $\mathcal{V}=\left\{\boldsymbol{w}_{1}, \boldsymbol{w}_{2}, \ldots, \boldsymbol{w}_{2^{B_{n}}}\right\}$, the receiver selects for the $n$th subcarrier,

$$
\begin{aligned}
\hat{\boldsymbol{v}}_{n} & =\arg \max _{\boldsymbol{w} \in \mathcal{V}} \log \left(1+\rho \boldsymbol{w}^{\dagger} \boldsymbol{H}_{n} \boldsymbol{H}_{n}^{\dagger} \boldsymbol{w}\right) \\
& =\arg \max _{\boldsymbol{w} \in \mathcal{V}} \boldsymbol{w}^{\dagger} \boldsymbol{H}_{n} \boldsymbol{H}_{n}^{\dagger} \boldsymbol{w}
\end{aligned}
$$

and the associated capacity for the $n$th subcarrier

$$
C_{n}=E_{\boldsymbol{H}_{n}, \mathcal{V}} \log \left(1+\rho \hat{\boldsymbol{v}}_{n}^{\dagger} \boldsymbol{H}_{n} \boldsymbol{H}_{n}^{\dagger} \hat{\boldsymbol{v}}_{n}\right)
$$

where the expectation is over both distribution of $\boldsymbol{H}_{n}$ and $\mathcal{V}$. This quantization has to be performed for the beamforming vector of each subcarrier. Hence, the required total number of feedback bits increases linearly with the number of subcarriers, which is usually large, e.g., 512. In this work, we would like to propose the quantization scheme that maximizes the sum capacity for a given available feedback bits.

\section{Clustering OF SUbCARriers}

Since the number of channel taps is much lower than the number of subcarriers $(L \ll N)$, adjacent subcarriers are highly correlated. Thus, the optimal transmit beamforming vectors for those subcarriers are very similar. To reduce a number of quantization bits for the beamforming vectors, we propose to group nearby subcarriers into a cluster and use the same quantized beamformer for all subcarriers in the same cluster. For each cluster, the transmit beamformer of the center subcarrier or next to the center is used for all subcarriers. Thus, the quantized beamformer is best matched to the center subcarrier and is progressively less matched to subsequent subcarriers. Let $T$ be the number of subcarriers in each cluster. We assume that each cluster consists of even number of subcarriers. Hence, the sum capacity of the first cluster is given by

$$
\sum_{q=0}^{T-1} C_{q}=\sum_{q=0}^{T-1} E \log \left(1+\rho \hat{\boldsymbol{v}}_{\frac{T}{2}}^{\dagger} \boldsymbol{H}_{q} \boldsymbol{H}_{q}^{\dagger} \hat{\boldsymbol{v}}_{\frac{T}{2}}\right) .
$$

As the cluster size increases, the capacity loss due to mismatching beamformer grows. However, the number of quantization bits we are saving also increases.

Let $K=N / T$ be the total number of clusters and $K$ is assumed to be a whole number. With this proposed clustering, the sum capacity averaged over subcarriers is given by

$$
\bar{C}=\frac{1}{N} \sum_{k=1}^{K} \sum_{q=(k-1) T}^{k T-1} E \log \left(1+\rho \hat{\boldsymbol{v}}_{k \frac{T}{2}}^{\dagger} \boldsymbol{H}_{q} \boldsymbol{H}_{q}^{\dagger} \hat{\boldsymbol{v}}_{k \frac{T}{2}}\right) .
$$

Assuming total number of feedback bits $B$, the common beamforming vector in each cluster is quantized with $B / K$ bits. If $K$ is large, the transmit beamforming vector is updated very often throughout OFDM band, but has a large quantization error. On the other hand, if $K$ is small, the quantized transmit beamforming vector is updated less often, but with more accuracy. Thus, there is a performance tradeoff over the number of clusters $K$ or the cluster size $T$. We would like to determine the optimal cluster size that maximizes the sum capacity as follows

$$
T^{*}=\arg \max _{\substack{1 \leq T \leq N \\ T \in \mathbb{Z}}} \bar{C} .
$$

To analyze $\bar{C}$, we start with the inner product $\hat{\boldsymbol{v}}_{p}^{\dagger} \boldsymbol{H}_{r} \boldsymbol{H}_{r}^{\dagger} \hat{\boldsymbol{v}}_{p}$. With unlimited feedback $(B \rightarrow \infty)$, the quantized beamformer is matched to the channel

$$
\hat{\boldsymbol{v}}_{p} \rightarrow \hat{\boldsymbol{v}}_{p}^{\infty}=\boldsymbol{H}_{p} /\left\|\boldsymbol{H}_{p}\right\|
$$


Thus,

$$
E\left[\left(\hat{\boldsymbol{v}}_{p}^{\infty}\right)^{\dagger} \boldsymbol{H}_{p+q} \boldsymbol{H}_{p+q}^{\dagger} \hat{\boldsymbol{v}}_{p}^{\infty}\right]=E\left[\frac{\left|\boldsymbol{H}_{p}^{\dagger} \boldsymbol{H}_{p+q}\right|^{2}}{\left\|\boldsymbol{H}_{p}\right\|^{2}}\right] .
$$

With property of DFT and some algebraic manipulation, we can show that

$$
\begin{aligned}
E\left|\boldsymbol{H}_{p}^{\dagger} \boldsymbol{H}_{p+q}\right|^{2} & =E\left|\sum_{m=1}^{M} \boldsymbol{D}_{p}^{\dagger} \boldsymbol{h}_{m}^{*} \boldsymbol{h}_{m}^{T} \boldsymbol{D}_{p+q}\right|^{2} \\
& =\frac{M^{2}}{L^{2}}\left(\frac{\sin \frac{L \pi q}{N}}{\sin \frac{\pi q}{N}}\right)^{2}
\end{aligned}
$$

and

$$
E\left\|\boldsymbol{H}_{p}\right\|^{2}=E\left|\sum_{m=1}^{M} \boldsymbol{D}_{p}^{\dagger} \boldsymbol{h}_{m}^{*} \boldsymbol{h}_{m}^{T} \boldsymbol{D}_{p}\right|=M .
$$

Using (14) and (15), we approximate that

$$
E\left[\left(\hat{\boldsymbol{v}}_{p}^{\infty}\right)^{\dagger} \boldsymbol{H}_{p+q} \boldsymbol{H}_{p+q}^{\dagger} \hat{\boldsymbol{v}}_{p}^{\infty}\right] \approx \frac{M}{L^{2}}\left(\frac{\sin \frac{L \pi q}{N}}{\sin \frac{\pi q}{N}}\right)^{2} .
$$

Given that $q=0$ and $B / K$ quantization bits, it was shown by [13] that

$$
\frac{E\left[\left(\hat{\boldsymbol{v}}_{p}\right)^{\dagger} \boldsymbol{H}_{p} \boldsymbol{H}_{p}^{\dagger} \hat{\boldsymbol{v}}_{p}\right]}{E\left[\left(\hat{\boldsymbol{v}}_{p}^{\infty}\right)^{\dagger} \boldsymbol{H}_{p} \boldsymbol{H}_{p}^{\dagger} \hat{\boldsymbol{v}}_{p}^{\infty}\right]}=1-2^{B / K} \beta\left(2^{B / K}, \frac{M}{M-1}\right)
$$

$$
\triangleq \gamma(B / K)
$$

where $\beta(x, y)=\frac{\Gamma(x) \Gamma(y)}{\Gamma(x+y)}$ is the beta function and the gamma function $\Gamma(x)=\int_{0}^{\infty} t^{x} \mathrm{e}^{-t} \mathrm{~d} t$. Based on our simulation results, we find that similar relationship as in (17) also applies when $q \neq 0$. Thus, we approximate that

$$
E\left[\hat{\boldsymbol{v}}_{p}^{\dagger} \boldsymbol{H}_{p+q} \boldsymbol{H}_{p+q}^{\dagger} \hat{\boldsymbol{v}}_{p}\right] \approx \gamma(B / K) \frac{M}{L^{2}}\left(\frac{\sin \frac{L \pi q}{N}}{\sin \frac{\pi q}{N}}\right)^{2} .
$$

Applying Jensen's inequality and substituting (19) into (9), we have

$$
\bar{C} \lesssim \frac{1}{T} \sum_{q=0}^{T-1} \log \left(1+\rho \gamma(B / K) \frac{M}{L^{2}}\left(\frac{\sin \frac{L \pi\left(q-\frac{T}{2}\right)}{N}}{\sin \frac{\pi\left(q-\frac{T}{2}\right)}{N}}\right)^{2}\right) .
$$

We would like to maximize this approximate upper bound on sum capacity over cluster size $T$ for given total feedback $B$. Thus,

$$
\begin{aligned}
& \tilde{T}^{*}= \\
& \arg \max _{\substack{1 \leq T \leq N \\
T \in \mathbb{Z}}} \frac{1}{T} \sum_{q=0}^{T-1} \log \left(1+\rho \gamma(B / K) \frac{M}{L^{2}}\left(\frac{\sin \frac{L \pi\left(q-\frac{T}{2}\right)}{N}}{\sin \frac{\pi\left(q-\frac{T}{2}\right)}{N}}\right)^{2}\right) .
\end{aligned}
$$

Solving (21) requires integer optimization for which there are many tools available, and is much easier than solving for $T^{*}$ in (10) where numerical simulations are needed. In the next section, we will show that $\tilde{T}^{*}$ and the corresponding $\tilde{K}^{*}=$ $N / \tilde{T}^{*}$ can predict $T^{*}$ in (10) and $K^{*}$, respectively, very well.

\section{NumericAl Results}

In Fig 1, we plot the average capacity (9) obtained by simulations and the approximate capacity bound (20) with $B / M$ for different number of channel taps. We note that both increase with the number of feedback bits as expected and that both give the same performance trend. Also the gap between the simulation result and approximate upper bound narrows when the number of channel taps is large or the channel is heavily frequency-selective. In this example, subcarriers are divided into 8 clusters $(K=8)$. As the channel becomes more frequency selective (larger $L$ ), subcarriers are less correlated. Thus, updating the beamformer for every 8 subcarriers is not sufficient and the capacity decreases as shown.

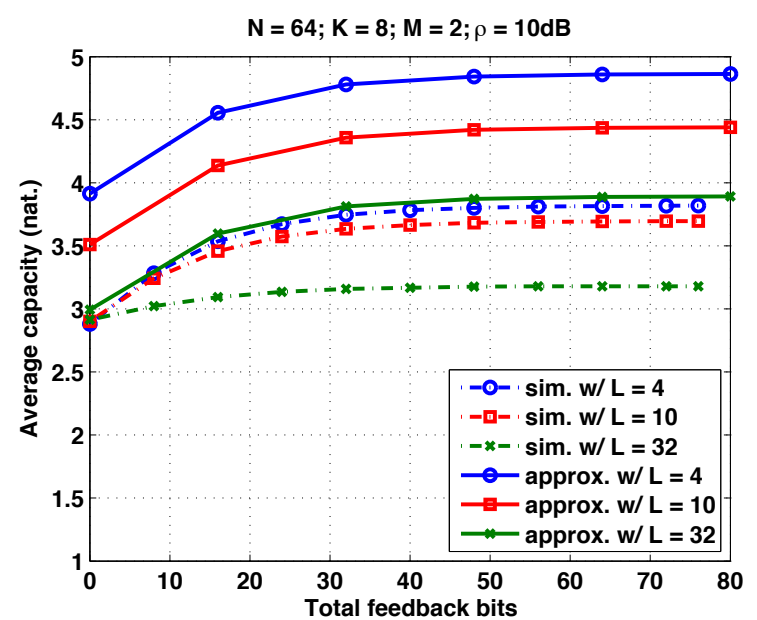

Fig. 1. A sum capacity is shown with the total number of feedback bits $B$ for $N=64, K=8, M=2$, and $\rho=10 \mathrm{~dB}$.

Fig. 2 shows the sum capacity with the number of clusters $K$ from both analytical approximation and simulation. Given a constraint on the number of feedback bits $B=48$, selecting the optimal number of clusters, which is shown by simulation result, may perform $50 \%$ better than not clustering $(K=N)$. We note that only cluster size that gives an integer number of clusters is plotted. Comparing the analytical approximation and simulation results, we observe that the gap is quite substantial, however, the analytical result still can accurately predict the optimal $K$. For a flat fading channel $(L=1)$, all subcarrier gains are the same and thus, the optimal $K^{*}=1$. For frequency selective fading $(L>1)$, subcarriers are less correlated and the optimal $K^{*}$ increases with $L$.

In Fig. 3, the optimal number of clusters $\tilde{K}^{*}$ computed from (21) is plotted with different number of channel taps. We see that $\tilde{K}^{*}$ increases with $L$. As channel becomes more frequency selective, the number of clusters should increase as well. The optimal $\tilde{K}^{*}$ also depends on the total feedback bits. In the figure shown, the number of clusters for $B=128$ is larger than that for $B=64$. Larger amount of feedback allows more clusters with quantization error essentially unchanged.

Fig. 4 shows $\tilde{K}^{*}$ with $B$ and $L$. For flat fading, the optimal number of cluster $\tilde{K}^{*}$ is always one regardless of available feedback bits. For frequency selectivity, $\tilde{K}^{*}$ increases with 


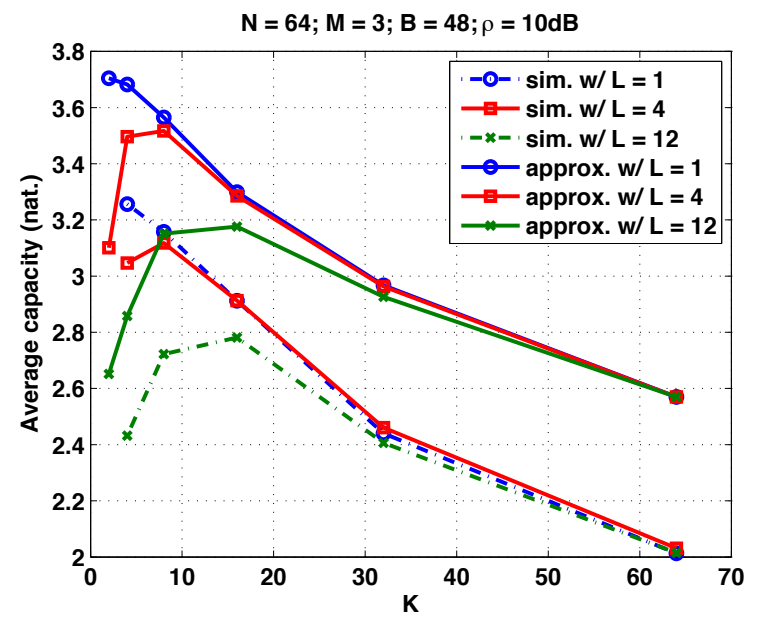

Fig. 2. Sum capacity is shown with the number of clusters $K$ and different number of channel taps $L$ for $N=64, M=3, B=48$ and $\rho=10 \mathrm{~dB}$.

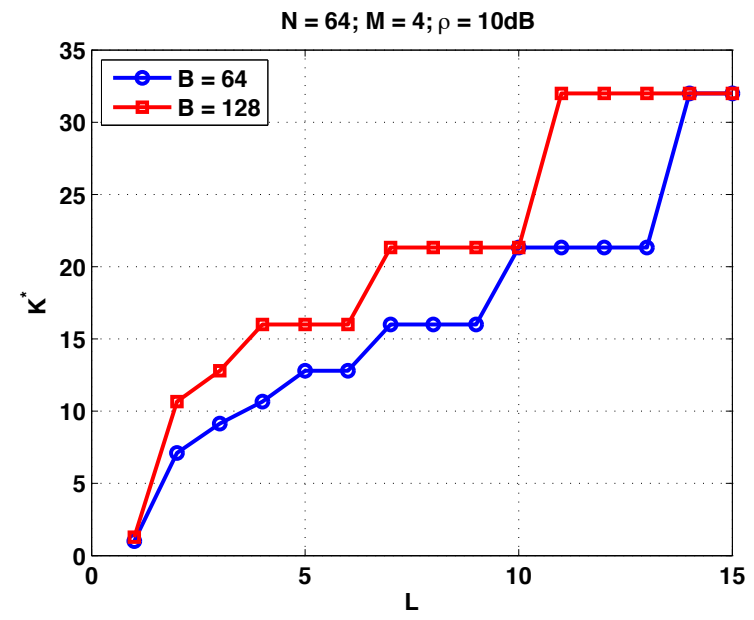

Fig. 3. The optimal $\tilde{K}^{*}$ is shown with $L$ and $B$ for $N=64, M=4$, and $\rho=10 \mathrm{~dB}$.

feedback bits. The larger the number of total feedback bits is, the larger the number of clusters should be.

\section{CONCLUSIONS}

In this work we have derived the approximation of the sum capacity upper bound, which can then be used to determine the optimal cluster size for given numbers of total feedback bits, channel taps, and transmit antennas. The optimal cluster size obtained from the analytical approximation is shown to accurately predict that from simulations and should be useful to system designers. The performance achieved by using the optimal cluster size is substantial when compared with selecting arbitrary size.

Here our analysis contains some crude approximations and hence, the gap between analytical and simulation results in some regime can be large. Refining the approximations is challenging and will be our future work. Moreover, we intend to extend our results to MIMO-OFDM and multiuser channels.

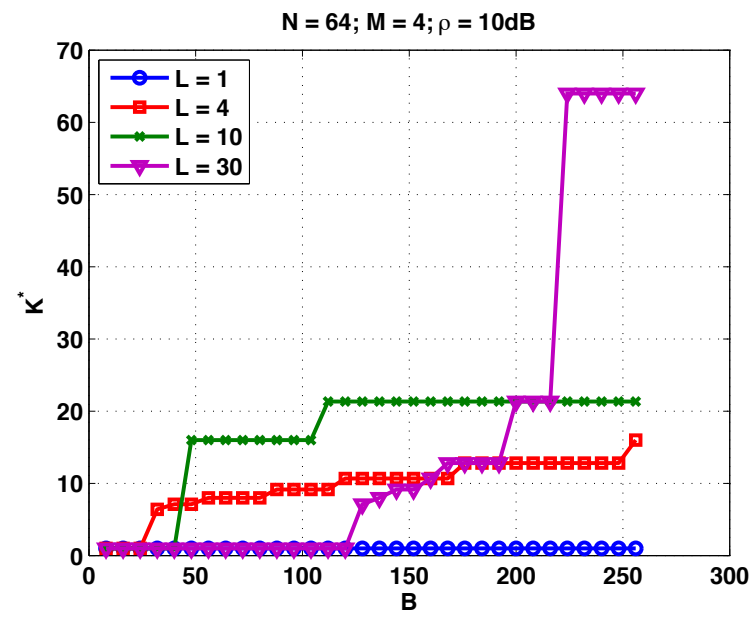

Fig. 4. The optimal $\tilde{K}^{*}$ is shown with $L$ for $N=64, M=4$, and $\rho=10$ dB.

\section{REFERENCES}

[1] İ. E. Telatar, "Capacity of multi-antenna Gaussian channels," European Trans. on Telecommun., vol. 10, pp. 585-595, Nov. 1999.

[2] H. Bolcskei and E. Zurich, "MIMO-OFDM wireless systems: Basics, perspectives, and challenges," IEEE Trans. Wireless Commun., vol. 13, no. 4, pp. 31-37, Aug. 2006.

[3] T. K. Y. Lo, "Maximum ratio transmission," IEEE Trans. Commun., vol. 47, no. 10, pp. 1458-1461, Oct. 1999.

[4] D. J. Love and R. W. Heath, Jr., "Grassmannian beamforming for multiple-input multiple-output wireless systems," IEEE Trans. Inf. Theory, vol. 49, no. 10, pp. 2735-2745, Oct. 2003.

[5] K. K. Mukkavilli, A. Sabharwal, E. Erkip, and B. Aazhang, "On beamforming with finite rate feedback in multiple antenna systems," IEEE Trans. Inf. Theory, vol. 49, no. 10, pp. 2562-2579, Oct. 2003.

[6] W. Santipach and M. L. Honig, "Capacity of a multiple-antenna fading channel with a quantized precoding matrix," IEEE Trans. Inf. Theory, vol. 55, no. 3, pp. 1218-1234, Mar. 2009.

[7] D. J. Love, R. W. Heath, Jr., V. K. N. Lau, D. Gesbert, B. D. Rao, and M. Andrews, "An overview of limited feedback in wireless communication systems," IEEE J. Sel. Areas Commun., vol. 26, no. 8, pp. 1341-1365, Oct. 2008.

[8] H. Z. Ye, V. Stolpaman, and N. van Waes, "A reduced CSI feedback approach for precoded MIMO-OFDM system," IEEE Trans. Wireless Commun., vol. 6, no. 1, pp. 55-58, Jan. 2007.

[9] J. Choi, B. Mondal, and R. W. Heath, Jr., "Interpolation based unitary precoding for spatial multiplexing MIMO-OFDM with limited feedback," IEEE Trans. Signal Process., vol. 54, no. 12, pp. 4730-4740, Dec. 2006.

[10] T. Pande, D. J. Love, and J. V. Krogmeier, "Reduced feedback MIMOOFDM precoding and antenna selection," IEEE Trans. Signal Process., vol. 55, no. 5, pp. 2284-2293, May 2007.

[11] R. Grunheid, E. Bolinth, and H. Rohling, "A blockwise loading algorithm for the adaptive modulation technique in OFDM system," in Proc. IEEE Vehicular Technology Conf. (VTC-Fall), vol. 2, Oct. 2001, pp. 948-951.

[12] D. J. Love, R. W. Heath, Jr., W. Santipach, and M. L. Honig, "What is the value of limited feedback for MIMO channels?" IEEE Commun. Mag., vol. 42, no. 10, pp. 54-59, Oct. 2004.

[13] C. K. Au-Yeung and D. J. Love, "On the performance of random vector quantization limited feedback beamforming in a MISO system," IEEE Trans. Wireless Commun., vol. 6, no. 2, pp. 458-462, Feb. 2007. 